\title{
Dendroecologia de Gymnanthes klotzschiana Müll. Arg. em um remanescente de Floresta Ombrófila Mista Aluvial no Sul do Brasil
}

\author{
Dendroecology of Gymnanthes klotzschiana Müll. Arg. in a remnant area of \\ Alluvial Mixed Ombrophilous Forest, Southern Brazil
}

\author{
Tomaz Longhi-Santos ${ }^{I}$, Franklin GalvãoI, Paulo Cesar Botosso ${ }^{\mathrm{II}}$, \\ Kelly Geronazzo Martins ${ }^{\text {III }}$
}

\begin{abstract}
Resumo
Em um remanescente de Floresta Ombrófila Mista Aluvial, foram abatidas 56 árvores adultas de Gymnanthes klotzschiana, árvore típica e representativa desta formação e popularmente conhecida como branquilho, às margens do rio Barigui, em Araucária - PR, o qual teve o curso de seu leito retificado na década de 1960. Considerando-se as técnicas e premissas básicas para estudos dendrocronológicos, os discos foram secos, polidos e os anéis de crescimento identificados e demarcados, para a construção de séries cronológicas e sua correlação com variáveis climáticas/ambientais. O presente trabalho tem por objetivo verificar se: (i) Gymnanthes klotzschiana apresenta características potenciais para estudos dendroecológicos; (ii) a espécie responde a estímulos ambientais e intervenções antrópicas; (iii) a estrutura etária das árvores estudadas é compatível com a ocorrência de distúrbios antrópicos na região. A análise dos resultados reforça a potencialidade da espécie em estudos dendroecológicos, considerando a sincronia dos dados e a sensibilidade às variáveis climáticas. Além disso, a idade máxima constatada das árvores analisadas é próxima ao final do ciclo de extração da madeira utilizada pelas embarcações a vapor nesta região, que preponderaram até meados da década de 1950.
\end{abstract}

Palavras-chave: Anéis de crescimento; Respostas ambientais; Intervenções antrópicas; Planície de inundação

\begin{abstract}
In a remnant area of Alluvial Ombrophylous Forest, 56 adult trees of Gymnanthes klotzschiana, a typical and representative tree of this formation and popularly known as "branquilhos", were slaughtered on the banks of the Barigui River, in Araucária, PR state, which the watercourse was rectified in the 1960s. Following the techniques for dendrochronological studies, the discs were dried, polished and the growth rings identified and marked, for the construction of chronological series and their correlation with climatic/environmental variables. This study aims to verify, if (i) Gymnanthes klotzschiana presents potential characteristics for dendroecological studies; (ii) the species responds to environmental stimuli and anthropogenic disturbances (iii) the age structure of the studied trees is compatible with environmental disturbances, documented for the region. The analysis of the results reinforces the potentiality of the species in dendroecological studies, considering the synchrony of the data and the sensitivity to the climatic variables. In addition, the maximum tree age corresponds to the end of the harvest intervention in this region, which prevailed until the middle of 1950s.
\end{abstract}

Keywords: Tree rings; Environmental responses; Anthropogenic disturbances; Floodplain

\footnotetext{
Engenheiro Florestal, Dr., Professor do Departamento de Ciências Florestais, Setor de Ciências Agrárias, Universidade Federal do Paraná, Av. Pref. Lothário Meissner, 632, CEP 80210-170, Curitiba (PR), Brasil. tomazlonghi@gmail.com (ORCID: 0000-0001-9700-2843) / fgalvao@ufpr.br (ORCID: 0000-0002-3492-5090)

II Engenheiro Florestal, Dr., Pesquisador da Embrapa Florestas, Estrada da Ribeira, km 111, s/n, CEP 83411-000, Colombo (PR), Brasil. paulo.botosso@embrapa.br (ORCID: 0000-0001-5361-2543)

III Bióloga, Dr ${ }^{\mathrm{a}}$., Professora do Departamento de Engenharia Ambiental, Setor de Ciências Agrárias e Ambientais, Universidade Estadual do CentroOeste, PR 153, Km 7, CEP 84500-000, Irati (PR), Brasil. kellygm77@gmail.com (ORCID: 0000-0002-0447-4444)
} 


\section{Introdução}

As características climáticas, geomorfológicas e pedológicas de um local são uns dos diversos fatores responsáveis pelo estabelecimento e desenvolvimento de uma comunidade vegetal (BAZZAZ, 1979; STEVEN, 1991; WALKER; WARDLE, 2014). As espécies que compõem estas comunidades e suas interações com os meios biótico e abiótico são resultado das oportunidades, restrições e facilitações de cada momento (WHITMORE, 1989).

Uma modificação ou alteração ambiental, natural ou antrópica, requer uma demanda de energia, diferente do habitual, para que as espécies se adaptem, tanto para seu estabelecimento quanto para retomada de crescimento (COX; MOORE, 2009). Esta adaptação é evidente no ritmo e padrão de crescimento das espécies, podendo ser registrada nos anéis de crescimento formados em muitas espécies lenhosas (ROZENDAAL; ZUIDEMA, 2010).

Durante muito tempo, a existência de anéis de crescimento anuais ou sazonais em regiões tropicais e subtropicais foi desacreditada (ROZENDAAL; ZUIDEMA, 2010), uma vez que se esperava que essas camadas de crescimento fossem formadas, quase que exclusivamente, por variações bem marcadas de temperatura e/ou precipitação, a exemplo dos ecossistemas de clima temperado (GRAU et al., 2003).

A existência de espécies com anéis de crescimento formados com periodicidade conhecida abriu a possibilidade de estudos dendrocronológicos e dendroecológicos em diferentes ambientes no hemisfério Sul (VILLALBA et al., 1998). No Brasil, os estudos com anéis de crescimento se intensificaram a partir da década de 1980, principalmente na região amazônica, nas florestas inundáveis (TOMAZELLO-FILHO et al., 2009). No Sul do Brasil (florestas subtropicais), Seitz e Kanninen (1989) foram os pioneiros nos estudos dendrocronológicos ao investigarem os anéis de crescimento de Araucaria angustifolia.

Hoje, em todas as regiões do Brasil são conduzidos trabalhos dendrocronológicos e dendroecológicos, com o intuito de caracterizar a idade dos remanescentes florestais, desenvolver cronologias regionais, buscar informações sobre a dinâmica florestal dessas áreas e compreender a autoecologia das espécies que as compõem. Os esforços se concentram, sobretudo, em áreas de floresta ombrófila densa na região amazônica (SCHÖNGART et al., 2002; WORBES, 2002; DÜNISCH et al., 2003; BONINSEGNA et al., 2009; TOMAZELO-FILHO et al., 2009; JIMÉNEZ, 2011), mas, aos poucos, se disseminam por todas as outras unidades fitogeográficas no país: Floresta Ombrófila Mista, na região Sul (SEITZ; KANNINEN, 1989; OLIVEIRA, 2007); Floresta Ombrófila Densa, na região Sudeste (CALLADO et al., 2001); Floresta Estacional Decidual, na região Sul (RIGOZO et al., 2004); Floresta Estacional Semidecidual na região Sudeste (LISI et al., 2008).

Em áreas ocupadas pela Floresta Ombrófila Mista, característica da região Sul do Brasil, muitas espécies atendem às premissas para estudos dendrocronológicos e apresentam potencialidades na identificação de distúrbios naturais e antrópicos (OLIVEIRA, 2007). Assim, este estudo investigou os anéis de crescimento de Gymnanthes klotzschiana - uma espécie arbórea típica e predominante em Floresta Ombrófila Mista Aluvial (RODERJAN et al., 2002), chegando a recobrir mais de 60\% destas áreas (CARVALHO et al., 2016), que foi intensamente utilizada durante o período de navegação no rio Iguaçu, no Paraná, servindo de energia para os vapores que transportavam madeira e erva-mate (LONGHI-SANTOS, 2013) - com o intuito de verificar se: (i) G. klotzschiana apresenta características potenciais para estudo dendroecológicos; (ii) a espécie responde a estímulos ambientais e intervenções antrópicas; (iii) a estrutura etária das árvores estudadas é compatível com distúrbios ambientais, documentados para a região. 


\section{Material e métodos}

\section{Caracterização da área de estudo}

A área de estudo está inserida em um remanescente florestal, no município de Araucária - PR, localizado ao sul da Região Metropolitana de Curitiba (Figura 1), na porção centro-sul do primeiro planalto paranaense a $890 \mathrm{~m}$ s.n.m., à margem direita do rio Barigui, entre as coordenadas $25^{\circ} 35^{\prime} 13.21^{\prime \prime} \mathrm{S}$ e $49^{\circ} 20^{\prime} 48.79$ ” O.

A precipitação média anual na região de Curitiba, para o período de 1961 a 2001 (com base no período de dados disponibilizados pelo INMET), foi de aproximadamente $1460 \mathrm{~mm}$, com valores anuais menores que $600 \mathrm{~mm}$ (1985) e maiores que $2000 \mathrm{~mm}$ (1998), com verões chuvosos (dezembro a março) e invernos relativamente secos (junho a setembro), quando comparados às demais estações do ano, com a ocorrência recorrente de geadas. A temperatura média máxima para os meses mais quentes foi de $25,4^{\circ} \mathrm{C}$ e a média mínima para os meses mais frios de $10,3^{\circ} \mathrm{C}$ (Figura 1).

Figura 1 - Localização da área de estudo e o climatograma para a região (barras representam precipitação mensal e as linhas temperaturas máxima, média e mínima, construído com uma série histórica de dados da região de 1961-2001 - INMET).

Figure 1 - Location of the study area and climatogram for the region (bars represent the monthly precipitation and the lines the maximum, average and minimum temperatures, constructed with a historical series of data of the region from 1961-2001 - obtained from INMET).

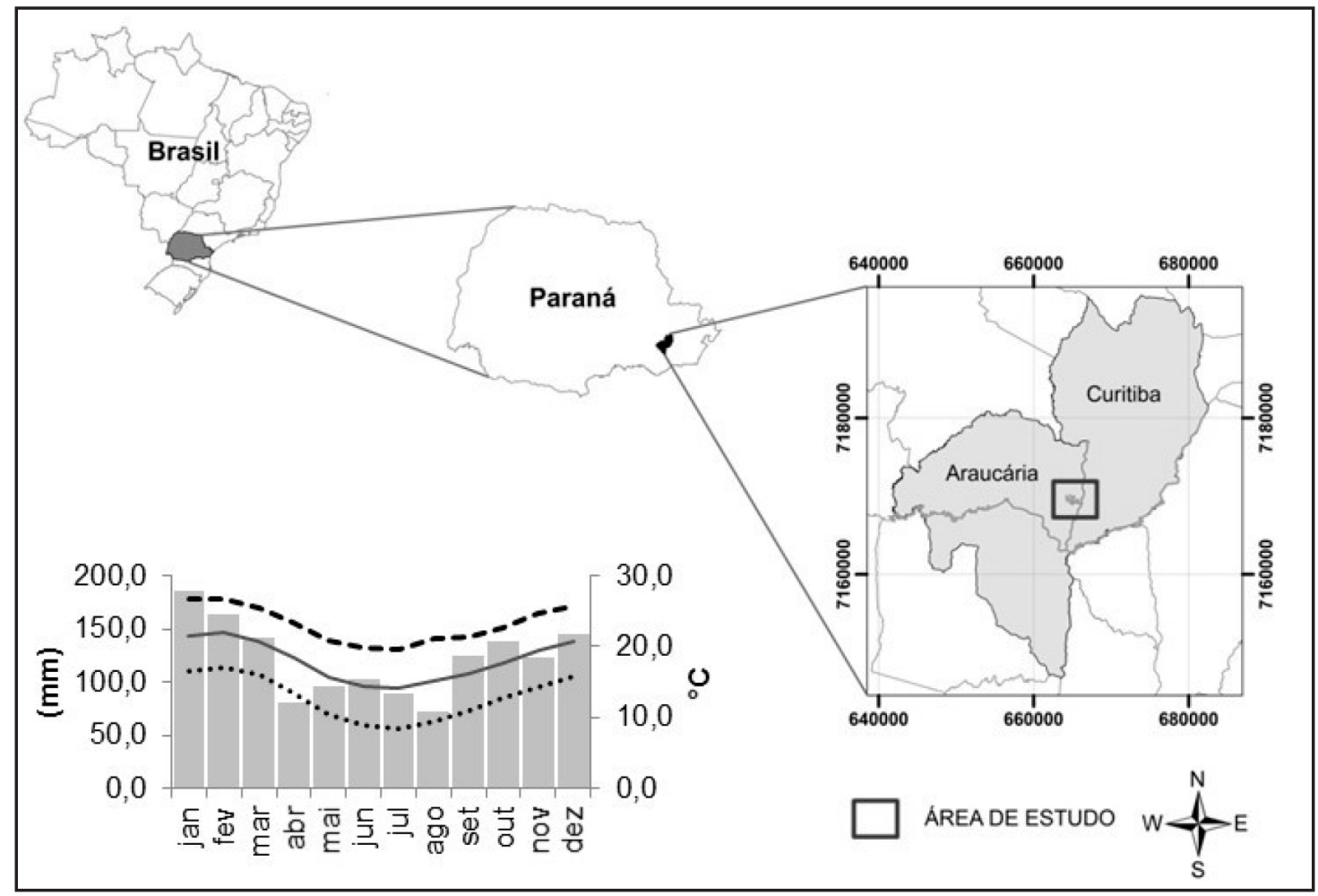

Fonte: Autores (2019)

O solo da área de estudo é de origem alúvio-coluvionar (influência direta da dinâmica fluvial), formado por sedimentos de fina granulometria, sendo classificado como Gleissolo Háplico 
(BARDDAL, 2002). Esta classe é formada por solos hidromórficos, mal ou muito mal drenados, submetidos à saturação hídrica permanente ou sazonal por ascensão do lençol freático ou por extravasamento do rio em períodos de cheias, tornando o ambiente altamente seletivo (EMBRAPA, 2006).

Com extensão aproximada de $66 \mathrm{~km}$, o rio Barigui drena até sua foz $279 \mathrm{~km}^{2}$ e é o principal afluente do rio Iguaçu, à margem direita (FILL et al., 2005). Com o aumento da frequência dos extravasamentos do rio, como medida de contenção para as constantes enchentes na cidade Curitiba (em decorrência do crescimento desordenado), na metade da década de 1960 e início da de 1970, o rio Barigui teve seu curso retificado, passando de um leito meandrante para um traçado muito mais retilíneo, para que tivesse maior vazão (LONGHI-SANTOS, 2013).

\section{Seleção da espécie e coleta de dados}

Com a construção da usina termoelétrica, UEG-Araucária, no ano de 2000, surgiu a necessidade da instalação de torres de transmissão para o escoamento da energia produzida. Previamente à construção de uma dessas torres, em um fragmento florestal, o Instituto Ambiental do Paraná (IAP) autorizou a instalação de uma parcela amostral de $200 \mathrm{~m}^{2}$ e a supressão da vegetação ali existente (o que viria a ser a área deste estudo).

No remanescente florestal, a Floresta Ombrófila Mista Aluvial recobre a área que está inserida na planície de inundação do rio Barigui. Nela ainda é possível encontrar boa parte das espécies típicas desta formação, citadas por Roderjan et al. (2002), como: Gymnanthes klotzschiana Müll.Arg., Allophyllus edulis (A.St.-Hil. et al.) Hieron. ex Niederl., Myrrhinium atropurpureum Schott, Schinus terebinthifolius Raddi, Blepharocalyx salicifolius (Kunth) O.Berg, espécies que estão entre as de maior valor de importância neste ambiente.

Popularmente conhecida como branquilho, Gymnanthes klotzschiana Müll.Arg. é uma arvoreta de 10 a 15 metros de altura e de 20 a $30 \mathrm{~cm}$ de diâmetro (SOUZA; LORENZI, 2005) que predomina nas florestas aluviais da região Sul do Brasil e com longevidade que supera 80 anos (STASIAK et al., 2009). Ocorre naturalmente de Minas Gerais até o Rio Grande do Sul, podendo ser encontrada também no Uruguai, Argentina e leste do Paraguai (CARVALHO, 2003).

O branquilho é uma espécie que possui camadas de crescimento distintas a pouco distintas, com a presença eventual de falsos anéis e de anéis descontínuos no lenho. Os verdadeiros e distintos anéis de crescimento são demarcados pelo maior achatamento e espessamento radial das paredes das fibras (RODRIGUES, 2005; COSMO, 2008; COSMO et al., 2010).

Assim, em uma parcela de 10 × $20 \mathrm{~m}\left(200 \mathrm{~m}^{2}\right)$ foram coletados discos de madeira a 1,30 m (DAP) de 56 árvores de branquilho, classificadas de acordo com sua posição fitossociológica em sub-bosque e dossel. Estes discos foram secos a temperatura ambiente e submetidos ao polimento superficial da seção transversal com uso de lixas de diferentes granulometrias (de 80 a 600 grãos $/ \mathrm{cm}^{2}$ ), com o intuito de melhor evidenciar os limites dos anéis de crescimento.

\section{Análise dos dados}

Em cada amostra do lenho foram traçados pelo menos quatro raios na direção casca-medula, considerando-se as particularidades de cada disco. Para a identificação e a marcação dos anéis de crescimento foi utilizado um microscópio estereoscópico Leica S8 APO e para as medições de largura dos anéis, empregou-se uma mesa de mensuração Velmex, acoplada a um sistema QuadraChek ${ }^{\circledR} 10$, com precisão de $2 \mu \mathrm{m}$.

Para verificar a acurácia da datação e a identificar possíveis falsos anéis, flutuações intraanuais e anéis ausentes, as séries temporais obtidas foram sincronizadas por árvore e entre árvores. A sincronização dos dados foi realizada com o auxílio do software COFECHA (HOLMES, 1983) e, pela análise gráfica dos incrementos correntes de cada série. O COFECHA correlaciona as séries cronológicas a partir da sobreposição de segmentos (pré-definidos) com uma média construída de todas as amostras (GRISSINO-MAYER, 2001), verificando estatisticamente esta relação, a qual serve como critério de controle para cada amostra analisada. Neste estudo, os dados foram analisados no COFECHA, utilizando-se um segmento de sobreposição (janela) de 20/10 anos para 
as árvores de dossel $(\mathrm{r}=0,515(\mathrm{p}<0,01)$ e 10/5 anos, para as árvores de sub-bosque $(\mathrm{r}=0,715(\mathrm{p}<0,01)$, em função da idade mínima verificada em cada grupo. Sugere-se que a janela de sobreposição seja, no mínimo, a metade da menor idade encontrada para as árvores amostradas (LONGHI-SANTOS, 2013).

Para a construção de uma cronologia local utilizou-se o Programa ARSTAN (COOK; HOLMES, 1984) o qual remove as tendências de crescimento biológico, não dependentes de sinais climáticos, transformando os incrementos correntes em índices (COOK; KAIRIUKSTIS, 1990). A qualidade da cronologia foi verificada pelo valor de intercorrelação na sincronia dos dados e de acordo com o índice EPS (Expressed Population Signal). O índice EPS indica o quão bem um conjunto finito de dados pode representar uma população baseada num número infinito de amostras (BRIFFA; JONES, 1990; PIRAINO et al., 2015). Assume-se que o índice EPS é adequado quando o valor alcançado é maior que 0,85 para todo o período avaliado (neste caso, o índice foi calculado para todas as amostras considerando-se uma janela de sobreposição de 10 e 5 anos). Este valor é apenas um guia (WIGLEY et al., 1984) e alguns trabalhos, nas regiões tropicais e subtropicais, já consideram a redução do valor deste índice (PIRAINO et al., 2015).

A cronologia obtida foi correlacionada (correlação de Pearson) às séries históricas de precipitação e temperatura, oriundas da estação meteorológica do Instituto Nacional de Meteorologia (INMET) para a cidade de Curitiba, a partir de dados disponibilizados para o período de 1961 a 2001.

\section{Resultados e discussão}

Das 56 árvores analisadas, 36 pertenciam ao dossel e 20 ao sub-bosque do fragmento estudado. Assim, conforme a Tabela 1, dos 36 branquilhos de dossel, 16 foram sincronizados e das 20 árvores de sub-bosque, 10 foram sincronizadas.

\section{Tabela 1 - Parâmetros considerados e resultados obtidos na avaliação da sincronia dos dados entre as árvores de Gymnanthes klotzschiana, com uso do programa COFECHA.}

Table 1 - Parameters and results obtained in the evaluation of the data synchrony among the Gymnanthes klotzschiana trees, using the COFECHA program.

\begin{tabular}{lccc}
\hline \multirow{2}{*}{ Parâmetros } & Dossel & Sub-bosque & Ambas \\
\cline { 2 - 4 } & $\begin{array}{c}1959-1999 \\
(41 \text { anos })\end{array}$ & $\begin{array}{c}1975-1999 \\
(25 \text { anos })\end{array}$ & $\begin{array}{c}1959-1999 \\
(41 \text { anos })\end{array}$ \\
\hline Período considerado & $16($ de 36$)$ & $10($ de 20$)$ & $20(13 \mathrm{D} ; 7 \mathrm{~S})$ \\
Número de árvores & 30 & 24 & 39 \\
Séries temporais & 734 & 456 & 866 \\
Anéis analisados & 0,442 & 0,497 & 0,472 \\
Intercorrelação $(\mathbf{r})$ & 0,515 & 0,715 & 0,515 \\
Ponto crítico* & 0,387 & 0,427 & 0,387 \\
Sensibilidade média $(\mathbf{s})$ & & & \\
*: probabilidade $(99 \%)$. & & & \\
& & &
\end{tabular}

Nem todas as árvores amostradas compõem as cronologias desenvolvidas, considerando a interação apresentada por cada uma na sincronização dos dados. Ainda que haja sincronia entre os raios de uma mesma árvore, quando analisados com outras amostras, este comportamento não é verificado. A seleção das melhores séries radiais temporais é uma estratégia adotada para evidenciar com maior confiança sinais e ruídos registrados nas cronologias (VILLALBA et al.,1998; OLIVEIRA et al., 2009; BRANDES et al., 2011; CATTANEO et al., 2013). 
Com o objetivo de estabelecer uma cronologia para a área de estudo, as análises posteriores contemplaram o conjunto de amostras provenientes de ambas as posições sociológicas (dossel e sub-bosque). Essa sincronia seguiu as mesmas tendências das análises por grupo, seja na intercorrelação ou na média de sensibilidade. Foram consideradas 20 árvores e 39 raios, sendo 13 árvores de dossel e 7 de sub-bosque (Figura 2).

Ainda que as intercorrelações estejam abaixo do ponto crítico para significância dos dados, a 99\% de probabilidade, os valores correspondem, em geral, aos esperados para espécies tropicais, quando comparados as intercorrelações verificadas para outras espécies da América do Sul (DÜNISCH et al., 2003; OLIVEIRA et al., 2009; BRANDES et al., 2011; COSTA et al., 2015).

Características intrínsecas à estrutura macroscópica do lenho de Gymnanthes klotzschiana em algumas porções dos discos analisados, como formação frequente de falsos anéis, anéis descontínuos e/ou pouco distintos, bem como a presença de fibras gelatinosas (MENNEGA, 2005; RODRIGUES, 2005; COSMO, 2008; COSMO et al., 2010), podem estar associadas às dificuldades de delimitação dos anéis de crescimento e, consequentemente, a baixas intercorrelações. Além disso, outros fatores climáticos/ambientais, como a recorrência de pulsos de inundação na área, podem interferir nas características anatômicas do lenho da espécie e, por sua vez, nos valores de intercorrelação.

Todavia, os resultados de sincronismo obtidos neste estudo não diferem, de forma considerável, dos verificados para outras espécies tropicais e subtropicais de diferentes unidades fitogeográficas no país, grupos ecológicos e padrões de decidualidade foliar (SEITZ; KANNINEN, 1989; Dünisch et al., 2003; Chagas, 2009; Oliveira et al., 2009; Rauber, 2010; Lobão, 2011; Andreacci, 2012; Rigozo et al., 2012; Longhi-Santos, 2013; Miranda, 2015).

Por outro lado, a média de sensibilidade (mean sensitivity) foi superior a 0,30 (GRISSINOMAYER, 2001), indicando que a variabilidade interanual pode estar relacionada à sensibilidade climática (FRITTS, 1971; 1976). Brandes et al. (2011), trabalhando com lianas leguminosas na Floresta Atlântica, encontraram valores médios de sensibilidade superiores aos do presente trabalho, enquanto Oliveira (2007), analisando os anéis de crescimento de Araucaria angustifolia, encontrou valores baixos ou intermediários para as suas cronologias.

Loomans (1993), reconstruindo pulsos de inundação no sul do estado de Ilinois (EUA), obteve média de sensibilidade de 0,221 e considerou o valor alto, por se tratar de uma área de pouca drenagem e de grande umidade. Considera que este resultado pode refletir o fato de a planície de inundação ser desigual, com bancos de sedimentos e regiões abaciadas, diferentes padrões de drenagem e de influência das enchentes. A exemplo das condições existentes na área de estudo, essas variações no terreno, ao longo da vida das plantas, podem, também, ter favorecido na formação de falsos anéis e/ou de anéis descontínuos.

Em alguns discos também foram verificadas máculas nas porções do lenho inicial, consideradas comuns para a espécie (RODRIGUES, 2005; COSMO et al., 2010). Grau et al. (2003), por sua vez, trata a presença de máculas e/ou de outras injúrias mecânicas, em espécies de ambientes aluviais, como resultantes do estresse fisiológico, mudanças no solo e na topografia, provocados pelos pulsos de inundação.

As evidências verificadas por Loomans (1993) são também comuns ao remanescente analisado, uma vez que são perceptíveis diferenças microtopográficas na área de estudo, bem como o acúmulo de sedimentos em diferentes porções do terreno, após cada pulso de inundação ou com a ascensão do lençol freático.

A frequência de pulsos de inundação e períodos prolongados de enchentes são respostas das características naturais (estrutura geológica e lineamentos) do padrão de um rio, do volume de precipitação da região, da geomorfologia e da vegetação que o envolve (JUNK et al., 1989). Por outro lado, a vegetação de planícies aluviais é particular e responde a alterações na dinâmica de um rio.

Em função do processo de retificação do leito do rio Barigui, um grande volume de resíduos foi depositado às margens do rio, formando diques laterais alçados, que acabam por controlá-lo. Se antes a vegetação estava submetida aos pulsos naturais de inundação e ao aporte de sedimentos e nutrientes recorrentes, com a retificação do curso d'água, passou a não receber mais essa contribuição, na mesma frequência e intensidade que antes desta intervenção.

Outro aspecto importante a ser considerado é o fato de que, apesar de conter os pulsos de inundação, os diques alçados também dificultam que o excesso de água, armazenado pela ascensão 
do lençol freático ou pelo escoamento das encostas, seja drenado de acordo com a vazão do rio. Este aspecto poderia prolongar o tempo de manutenção da saturação hídrica do solo e, eventualmente, criar condições anóxicas e/ou hipóxicas temporárias das raízes, o que comprometeria o crescimento das árvores (KOZLOWSKI, 1984; CRAWFORD; BRÄNDLE, 1996).

Figura 2 - Síntese da sincronização: a) Séries cronológicas sincronizadas de Gymnanthes klotzschiana; b) Índice de largura de anéis de crescimento; c) Índice EPS (Expressed Population Signal) d) Número de séries temporais (raios amostrais) utilizadas por período analisado.

Figure 2 - Summary of synchronization: a) Synchronized chronological series of Gymnanthes klotzschiana trees; b) Tree ring width index; c) EPS Index; d) Number of time series (sample rays) used per analyzed period.

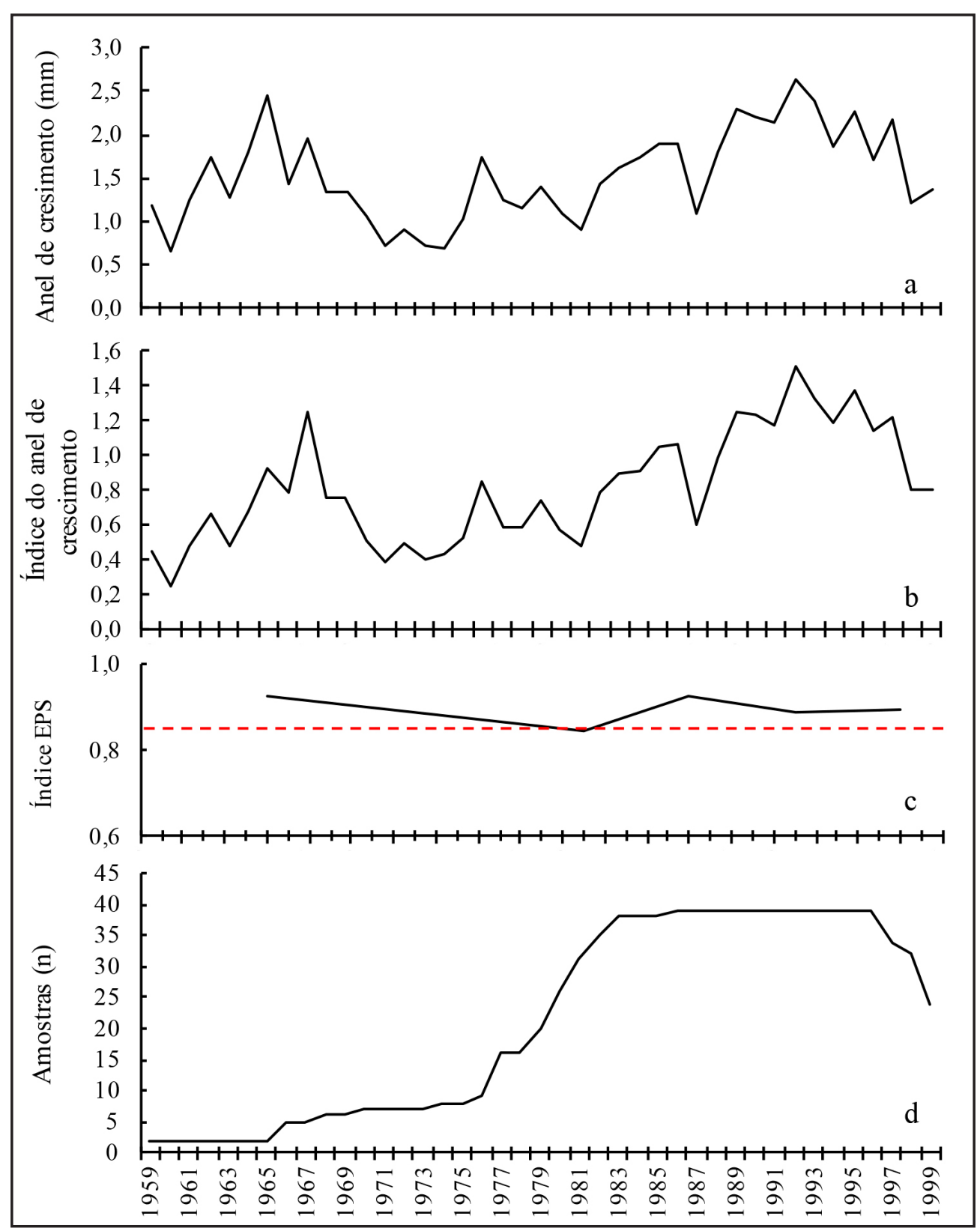

Fonte: Autores (2019) 
Gymnanthes klotzschiana é uma espécie pioneira (CARVALHO, 2003), logo, suas taxas de crescimento são maiores do que as de espécies consideradas não pioneiras (SWAINE; WITHMORE, 1988; TURNER, 2004), além do que sua longevidade tende a ser menor (MARTINS; RODRIGUES, 1999). Este comportamento pode auxiliar na compreensão das curtas séries temporais encontradas para a espécie neste estudo, além da variação entre os dados padronizados e a série média.

A extensão (idade) das séries temporais analisadas pode também estar relacionada ao período de maior extração madeireira ocorrida às margens do rio Iguaçu e de seus afluentes (rio Barigui, por exemplo), em um passado recente, visando alimentar as embarcações a vapor, utilizadas no transporte de mercadorias. Como a retirada de madeira para esta finalidade se encerrou em meados da década de 1950 (BACH, 2006), é possível que o fragmento desta área tenha sido explorado, se não, exclusivamente, para fornecer energia aos vapores, em alguma prática que necessitou da remoção da vegetação até por volta do final da década de 1950.

A disponibilidade hídrica, na forma de precipitação para ambientes aluviais, em tese, não é um fator limitante para o desenvolvimento da espécie (KANIESKI et al., 2013). Prova disso pode ser verificada na Figura 3, na qual apenas a precipitação do mês de janeiro do crescimento corrente é positiva e significativamente correlacionada com o crescimento. O mês de janeiro é, historicamente, o mês que registra o maior volume de chuvas para a região, mesmo período em que são, também, registradas as maiores temperaturas. Portanto, em se tratando da estação de maior crescimento, as plantas demandariam maior quantidade de água em seus processos fisiológicos, além do que, com altas temperaturas, aumentariam as perdas por respiração (LARCHER, 2004).

Oliveira (2007), Andreacci (2012) e Andreacci et al. (2014), quando estudaram espécies da Floresta Ombrófila Mista Montana, no Sul do Brasil, também encontraram correlações positivas, porém, não significativas, para o crescimento prévio ou corrente com a precipitação.

Figura 3 - Correlações entre séries de índice de largura dos anéis de crescimento de Gymnanthes klotzschiana e precipitação mensal acumulada. (Mês)p indica o período prévio à estação de crescimento e (Mês)c indica o período corrente. Colunas em preto indicam correlações estatisticamente significativas $(\mathbf{p}<0,05)$.

Figure 3 - Relationship between series of index of width of growth rings of Gymnanthes klotzschiana trees and accumulated monthly precipitation. (Month)p indicates the period previous to the growing season and (Month)c indicates the current period. Black columns indicate statistically significant results $(\mathrm{p}<0.05)$.

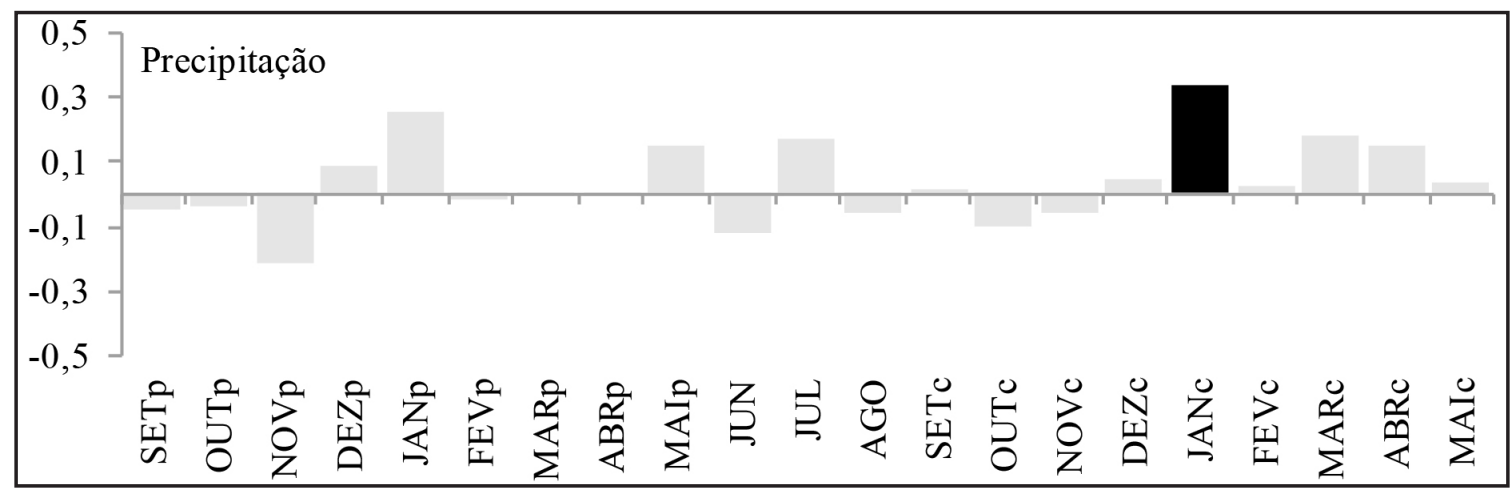

Fonte: Autores (2019)

Schweingruber (1996) considera a temperatura como um dos fatores limitantes de maior impacto no crescimento das árvores. Assim, as correlações estabelecidas com as temperaturas mínima, média e máxima apresentam maior interação com a série de índice de largura de anéis de crescimento de Gymnanthes klotzschiana, como pode ser observado na Figura 4. A média de temperatura mínima se correlaciona positiva e significativamente em boa parte dos meses, seja com relação ao crescimento prévio ou corrente. Isto indica que altas temperaturas mínimas 
influenciam positivamente na largura dos anéis de crescimento. Comportamento similar ao verificado por Andreacci (2012) e Andreacci et al. (2014), analisando árvores de Cedrela fissilis Vell. para a região de Curitiba - PR.

Figura 4 - Correlações entre séries de índice de largura dos anéis de crescimento das árvores de Gymnanthes klotzschiana com as médias mensais de temperatura (mínima, média e máxima). (Mês)p indica o período prévio à estação de crescimento e (Mês)c indica o período corrente. Colunas em preto indicam correlações estatisticamente significativas $(\mathbf{p}<\mathbf{0 , 0 5})$.

Figure 4 - Relationship between series of growth-ring width index of Gymnanthes klotzschiana trees with the monthly temperature averages (minimum, average and maximum). (Month)p indicates the previous to the growing season and (Month)c indicates the current period. Black columns indicate statistically significant results $(\mathrm{p}<0.05)$.

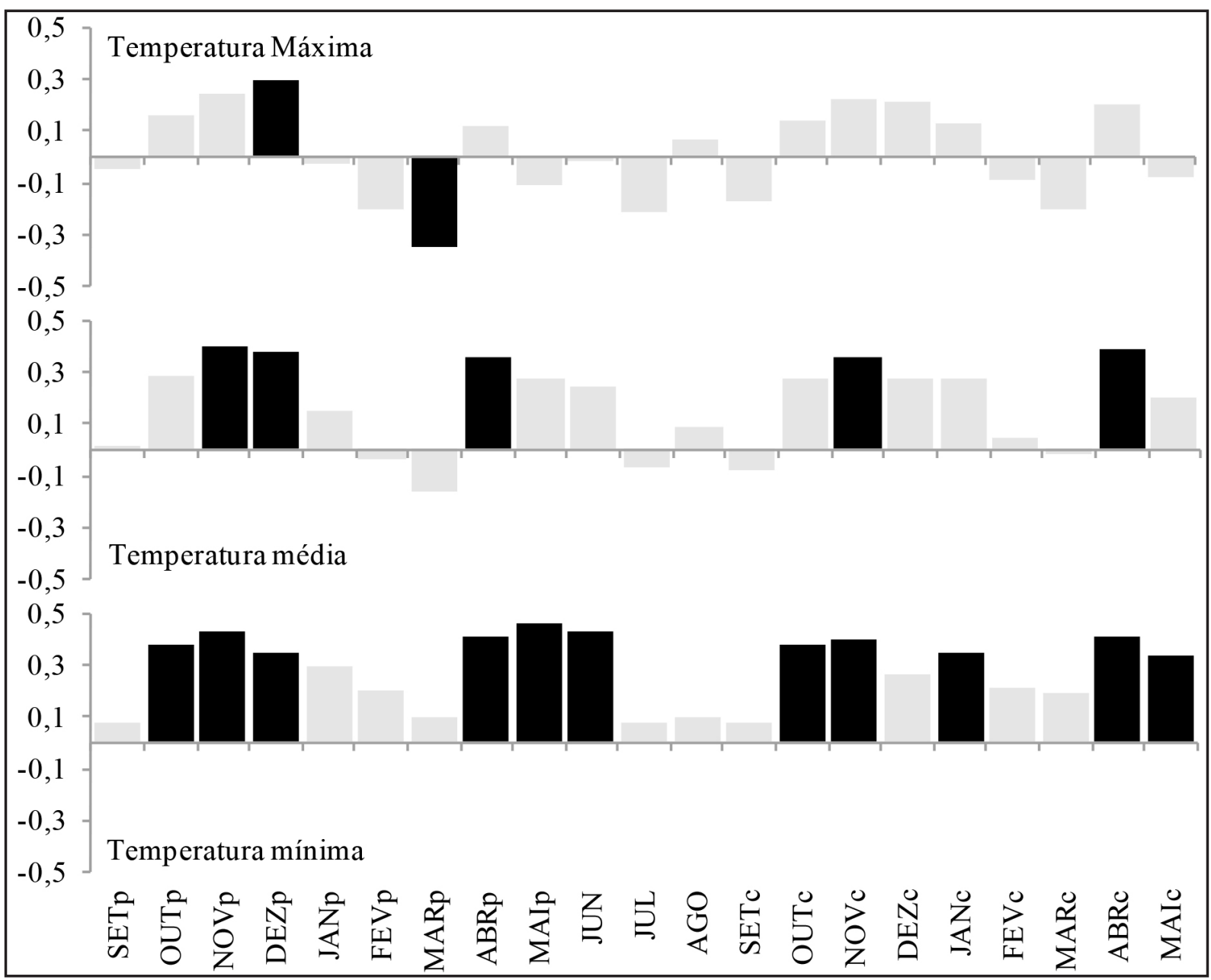

Fonte: Autores (2019)

Já para a temperatura máxima, as altas temperaturas do mês de março, prévio ao período de crescimento, apresentam correlação negativa e significativa com o crescimento, repetindo a tendência no período corrente, entretanto, sem significância estatística. Além disso, o período correspondente à retomada de crescimento das árvores, apresenta correlações positivas com a temperatura (mínima, média ou máxima), indicando a influência direta desta variável no crescimento de branquilho, também verificado por Kanieski et al. (2012). 


\section{Conclusões}

A sincronia dos dados das séries cronológicas de Gymnanthes klotzschiana (branquilho) apresentaram resultados que demonstram a existência de sinais comuns e a possibilidade de utilização da espécie para estudos dendroecológicos. Cabe ressaltar que os resultados apresentados estão dentro do esperado e verificado para espécies tropicais e subtropicais.

A média de sensibilidade para a cronologia proposta é considerada alta, indicando que a diferença na largura de anéis de um ano para outro está relacionada à sensibilidade às variações ambientais (pulsos de inundação e a retificação do leito do rio, por exemplo). Verificou-se que a temperatura, sobremaneira à mínima, pode ser considerada determinante no crescimento secundário das árvores, possivelmente como resultado da ativação e dormência do câmbio.

A idade das árvores analisadas é compatível com as informações documentadas e indícios de que o histórico de exploração da madeira desta espécie, nestes ambientes aluviais, tenha influência sobre a faixa etária das árvores amostras.

\section{Referências}

ANDREACCI, F. Atividade cambial, fenologia vegetativa e ritmo de crescimento de Cedrela fissilis Vell. em áreas de Florestas Ombrófilas Mista e Densa do estado do Paraná: aspectos dendrocronológicos e dendroecológicos. 2012. Dissertação(Mestrado em Botânica)-Universidade Federal do Paraná, Curitiba, 2012.

ANDREACCI, F. et al. Sinais climáticos em anéis de crescimento de Cedrela fissilis em diferentes tipologias de florestas ombrófilas do sul do brasil. Floresta, Curitiba, v. 44, n. 2, p. 323-332, abr./ jun. 2014.

BACH, A. M. Vapores. Ponta Grossa: Ed. UEPG, 2006. 493 p.

BARDDAL, M. L. Aspectos florísticos e fitossociológicos do componente arbóreo-arbustivo de uma floresta ombrófila mista aluvial - Araucária, PR. 2002. Dissertação (Mestrado em Engenharia Florestal) - Universidade Federal do Paraná, Curitiba, 2002.

BAZZAZ, F. A. The physiological ecology of plant succession. Annual Review of Ecology and Systematics, Palo Alto, v. 10, p. 351-371, nov. 1979.

BONINSEGNA, J. A. et al. Dendroclimatological reconstructions in South America: a review. Palaeogeography, Palaeoclimatology, Palaeoecology, Amsterdam, v. 281, n. 3/4, p. 210-228, aug. 2009.

BRANDES, A. F. N. et al. Dendrochronology of lianas of the Leguminosae family from the Atlantic Forest, Brazil. Trees, Berlin, v. 25, p. 133-144, feb. 2011.

BRIFFA, K. R.; JONES, P. D. Basic chronology statistics and assessment. In: COOK, E. R.; KAIRIUKSTIS, L. A. (ed.). Methods of Dendrochronology: applications in the environmental sciences. Dordrecht: International Institute for Applied Systems Analysis (IIASA); Kluwer Academic Publishers, 1990. p 137-152.

CALLADO, C. H. et al. Periodicity of growth rings in some flood-prone trees of the Atlantic rainforest in Rio de Janeiro. Trees, Berlin, v. 15, p. 492-497, dec. 2001.

CARVALHO, J. et al. Monospecific Dominance in An Alluvial Mixed Ombrophyllous Forest In Southern Brazil. Australian Journal of Basic and Applied Sciences, [s. l.], v. 10, n. 10, p. 38-44, jun. 2016

CARVALHO, P. E. R. Espécies arbóreas brasileiras. Colombo: EMBRAPA Florestas, 2003. v. 1, p. 247-253. 
CHAGAS, M. P. Caracterização dos anéis de crescimento e dendrocronologia de árvores de Grevillea robusta A. Cunn, Hovenia dulcisThunb., Persea americana Mill. Tabebuia pentaphylla Hemsl. eTerminalia catappa L. nos municípios de Piracicaba e Paulínia, SP. 2009. Dissertação (Mestrado em Recursos Florestais) - Universidade de São Paulo, Piracicaba, 2009.

CATTANEO, N. et al. Sex-related, growth-climate association of Araucaria angustifolia in the neotropical ombrophilous woodlands of Argentina. Dendrochronologia, Verona, v. 31, n. 3, p. 147-152, jan. 2013.

COOK, E. R.; HOLMES, R. L. Program ARSTAN user's manual. Tucson: The University of Arizona Press, 1984.

COOK, E. R.; KAIRIUKSTIS, L. A. Methods of dendrochronology: applications in the environmental science. Amsterdam: Kluwer, 1990.

COX, C. B.; MOORE, P. D. Biogeografia: uma abordagem evolucionária. Rio de Janeiro: LTC, 2009.

COSMO, N. L. Anatomia ecológica e crescimento do lenho de Gymnanthes klotzschiana (Baillon) Smith \&Downs, em diferentes condições geomorfológicas e pedológicas da planície do rio Iguaçu-PR. 2008. Dissertação (Mestrado em Engenharia Florestal) - Universidade Federal do Paraná, Curitiba, 2008.

COSMO, N. L. et al. Anatomia da madeira de Gymnanthes klotzschiana (Baillon) Smith \&Downs (Euphorbiaceae): aspectos funcionais e ecológicos. Acta Botanica Brasilica, Belo Horizonte, v. 24 , n. 3, p. 747-755, jul./set. 2010.

COSTA, M. S. et al. Growth analysis of five Leguminosae native tree species from a seasonal semidecidual lowland forest in Brazil. Dendrochronologia, Verona, v. 36, p. 23-32, 2015.

CRAWFORD, R. M. M.; BRANDLE, R. Oxygen deprivation stress in a changing environment. Journal of Experimental Botany, Oxford, v. 47, n. 2, p. 145-159, feb. 1996.

DÜNISCH, O. et al. Dendroecological investigations on Swietenia macrophylla King and Cedrela odorata L. (Meliaceae) in the Central Amazon. Trees, Berlin, v. 17, n. 3, p. 244-250, may 2003.

EMBRAPA. Sistema Brasileiro de Classificação de Solos. 2. ed. Rio de Janeiro: Centro Nacional de Pesquisa de Solos, 2006. 306 p.

FILL, H. D. et al. Balanço hídrico da bacia do Rio Barigüi, PR. RA'E GA, Curitiba, v. 9, n. 1, p. 59-67, 2005.

FRITTS, H. C. Dendroclimatology and dendroecology. Quaternary Research, Amsterdam, v. 1, n. 4, p. 419-449, dec. 1971.

FRITTS, H. C. Tree rings and climate. London: Academic Press, 1976.

GRAU, H. R. et al. Subtropical dendroecology-dating disturbances and forest dynamics in northwestern Argentina montane ecosystems. Forest Ecology and Management, Amsterdam, v. 177, n. 1, p. 131-143, abr. 2003.

GRISSINO-MAYER, H. D. Evaluating crossdating accuracy: a manual and tutorial for the computer program COFECHA. Tree-Ring Research, Tucson, v. 57, n. 2, p. 205-221, 2001.

HOLMES, L. R. Computer-assisted quality control in tree-ring dating and measurement. Treering Bulletin, Tucson, v. 43, p. 69-78, 1983.

JIMENEZ, J. A. G. Dendrocronología en el trópico: aplicaciones actuales y potenciales. Colombia forestal, Bogotá, v. 14, n. 1, p. 97-111, 2011.

JUNK, W. J. et al. The flood pulse concept in river-floodplain systems. Canadian Journal of Fishers and Aquatic, Ottawa, v. 106, p. 110-127, 1989. 
KANIESKI, M. R. et al. Crescimento Diamétrico de Blepharocalyx salicifolius em Remanescente de Floresta Ombrófila Mista Aluvial, Paraná. Floresta e Ambiente, Seropédica, v. 20, n. 2, p. 197206, abr.jun. 2013.

KANIESKI, M. R. et al. Influência da precipitação e da temperatura no incremento diamétrico de espécies florestais aluviais em Araucária-PR. Floresta e Ambiente, Seropédica, v. 19, n. 1, p. 17-25, jan./mar. 2012.

KOZLOWSKI, T. T. Responses of woody plants to flooding. In: KOZLOWSKI, T. T. (ed.). Flooding and Plant Growth. New York: Academic Press, 1984. p. 129-163.

LARCHER, W. Ecofisiologia vegetal. São Carlos: Rima Artes e Textos, 2004.

LISI, C. S. et al. Tree-ring formation, radial increment, periodicity and phenology of tree species from a seasonal semi-deciduous forest in Southeast Brazil. IAWA Journal, Leiden, v. 29, n. 2, p. 189-207, 2008.

LOBÃO, M. S. Dendrocronologia, fenologia, atividade cambial e qualidade do lenho de árvores de Cedrela odorata L., Cedrela fissilis Vell. E Schizolobium parahyba var. amazonicum Hub. ExDucke, no estado do Acre, Brasil. 2011. Tese (Doutorado em Recursos Florestais) Universidade de São Paulo, Piracicaba, 2011.

LONGHI-SANTOS, T. Dendroecologia de Sebastiania commersoniana (Baill.) L.B.Sm. \& Downs em um fragmento de Floresta Ombrófila Mista Aluvial, Paraná, Brasil. 2013. Dissertação (Mestrado em Engenharia Florestal) -Universidade Federal do Paraná, Curitiba, 2013.

LOOMANS, S. M. Flood reconstruction in Southern Illinois using tree rings. 1993. Thesis (Master of Science in Geography) - University of Illinois at Urbana-Champaign, Urbana, 1993.

MARTINS, S. V.; RODRIGUES, R. R. Produção de serapilheira em clareiras de uma floresta estacional semidecídua no Município de Campinas, SP. Revista Brasileira de Botânica, São Paulo, v. 22, p. 405-412, 1999.

MENNEGA, A. M. W. Wood anatomy of the subfamily Euphorbioideae: a comparison with subfamilies Crotonoideae and Acalyphoideae and the implications for circumscription of the Euphorbiaceae. IAWA Journal, Leiden, v. 26, n. 1, p. 1-68, 2005.

MIRANDA, B. P. Dendroecologia de Ilex microdonta Reissek e Drimys brasiliensis Miers em dois ambientes altomontanos da Serra do Mar, Paraná, Brasil. 2015. Dissertação (Mestrado em Engenharia Florestal) - Universidade Federal do Paraná, Curitiba, 2015.

OLIVEIRA, J. M. Anéis de crescimento de Araucaria angustifolia (Bertol.) O. Kuntze: bases de dendroecologia em ecossistemas subtropicais montanos no Brasil. 2007. Tese (Doutorado em Ecologia) - Universidade Federal do Rio Grande do Sul, Porto Alegre, 2007.

OLIVEIRA, J. M. et al. Climatic signals in tree-rings of Araucaria angustifolia in the southern Brazilian highlands. Austral Ecology, Carlton, v. 35, n. 2, p. 134-147, sep. 2009.

PIRAINO, S. et al. Geomorphological-related heterogeneity as reflected in tree growth and its relationships with climate of Monte Desert Prosopis flexuosa DC woodlands. Trees, Berlin, v. 29, n. 3, p. 903-916, fev. 2015.

RAUBER, R. C. Dendroecologia de Cedrela fissilis Vell. (Meliaceae) em um ecótono de Florestas Subtropicais Montanas no Brasil. 2010. Dissertação (Mestrado em Ciências) -Universidade Federal do Rio Grande do Sul, Porto Alegre, 2010.

RIGOZO, N. R. et al. Search for solar periodicities in tree-ring widths from Concórdia (S.C., Brazil). Pure and Applied Geophysics, Basel, v. 161, n. 1, p. 221-233, jan. 2004. 
RIGOZO, N. R. et al. Solar-Terrestrial Signal Record in tree ring width time series from Brazil. Pure and Applied Geophysics, Basel, v. 169, n. 12, p. 2181-2191, apr. 2012.

RODERJAN, C. V. et al. As unidades fitogeográficas do Estado do Paraná. Ciência \& Ambiente, Santa Maria, v. 24, p. 75-92, jan./jun. 2002.

RODRIGUES, T. T. Os efeitos do solo contaminado com petróleo na estrutura anatômica e estado nutricional do lenho jovem de Campomanesia xanthocarpa Berg (Myrtaceae) e Sebastiania commersoniana (Baillon) Smith \& Downs (Euphorbiaceae). 2005. Tese (Doutorado em Ciências Florestais) - Universidade Federal do Paraná, Curitiba, 2005.

ROZENDAAL, D. M. A.; ZUIDEMA, P. A. Dendroecology in the tropics: a review. Trees Structure and Function, Berlin, v. 25, n. 1, p. 3-16, fev. 2011.

SCHÖNGART, J. et al. Phenology and stem-growth periodicity of tree species in Amazonian floodplain forests. Journal of Tropical Ecology, Cambridge, v. 18, n. 4, p. 581-597, jul. 2002.

SCHWEINGRUBER, F. H. Tree-rings and environment dendroecology. Vienna: Swiss Federal Institute for Forest, Snow and Landscape Research, 1996. 609 p.

SEITZ, R. A.; KANNINEN, M. Tree ring analysis of Araucaria angustifolia in southern Brazil: preliminary results. IAWA Bulletin, Utrecht, v. 10, n. 2, p. 170-174, 1989.

SOUZA, V. C.; LORENZI, H. Botânica sistemática: guia ilustrado para identificação das famílias de Angiospermas da flora brasileira, baseado em APG II. Nova Odessa: Plantarum, 2005.

STASIAK, P. M. et al. Influência de Guadua aff. Paraguayana Döll (Poaceae) sobre o crescimento diametral do tronco de uma população de Sebastiania commersoniana (Baillon) Smith \& Downs. In: EVENTO DE INICIAÇÃO CIENTÍFICA DA EMBRAPA FLORESTAS, 8., 2009, Colombo. Anais [...] Colombo: EMBRAPA Florestas, 2009.

STEVEN, D. Experiments on mechanisms of tree establishment in old-field succesion: seedling emergence. Ecology, Washington, v. 72, n. 3, p. 1066-1075, jun. 1991.

SWAINE, M. D.; WHITMORE, T. C. On the definition of ecological species groups in tropical rain forests. Vegetatio, Dordrecht, v. 75, p. 81-86, may 1988.

TOMAZELLO FILHO, M. et al. Dendrocronología y dendroecología tropical: marco histórico y experiencias exitosas en los países de América Latina. Ecología en Bolivia, La Paz, v. 44, n. 2, p. 73-82, out. 2009.

TURNER, I. M. The ecology of trees in the tropical rainforest. Cambrigde: Cambridge University Press, 2004. 298 p.

VILLALBA, R. et al. Tree-ring evidence for long-term precipitation changes in subtropical South America. International Journal of Climatology, Chichester, v. 18 p. 1463-1478, nov. 1998.

WALKER, L. R.; WARDLE, D. A. Plant succession as an integrator of contrasting ecological time scales. Trends in Ecology \& Evolution, Amsterdam, v. 29, n. 9, p. 504-510, set. 2014.

WHITMORE, T. C. Canopy gaps and the two major groups of forest trees. Ecology, Washington, v. 70, n. 3 , p. 536-538, jun. 1989.

WIGLEY, T. M. L. et al. On the average value of correlated time series with application in dendroclimatology and hydrometereology. Journal Climate Applied Meteorology, Bonston, v. 23, p. 201-221, fev. 1984.

WORBES, M. One hundred years of tree-ring research in the tropics - a brief history and an outlook to future challenges. Dendrochronologia, Verona, v. 20, n. 1-2, p. 217-231, 2002. 